\title{
Assessment of lonizing Radiation Induced Dicentric Chromosome and Micronuclei in Human Peripheral Blood Lymphocytes for Preliminary Reconstruction of Cytogenetic Biodosimetry
}

\author{
M. Syaifudin ${ }^{1 *}$, Y. Lusiyanti ${ }^{1}$, S. Purnami ${ }^{1}$, Y.S Lee ${ }^{2}$ and C.M. Kang ${ }^{3}$ \\ ${ }^{I}$ Center for Technology of Radiation Safety and Metrology, National Nuclear Energy Agency \\ Jl. Lebakbulus Raya No. 49 Jakarta 12440, Indonesia \\ ${ }^{2}$ College of Pharmacy, Division of Life and Pharmaceutical Sciences, Ewha Womans University, Seoul, South Korea \\ ${ }^{3}$ Laboratory of Radiation Cytogenetics and Epidemiology, Korea Institute of Radiological and Medical Sciences (KIRAMS), \\ 215 Gongneung-Dong, Nowon-Ku, Seoul, South Korea
}

\section{ARTICLE INFO}

\section{Article history:}

Received 12 November 2015

Received in revised form 18 Mei 2016

Accepted 08 December 2016

\section{Keywords:}

Emergency irradiation exposure

Dicentric chromosome

Micronuclei

Biodosimetry

\begin{abstract}
A B S T R A C T
Cytological biodosimetry methodology has been widely used for determining and estimating the precise irradiation dose received by victims in the situation of emergency irradiation exposure. The aim of this study was to assess the gamma-ray induced dicentric chromosomes and micronuclei (MN) in peripheral blood lymphocytes for preliminary reconstruction of cytogenetic biodosimetry. The study was performed by exposing blood samples taken from seven healthy donors to gamma rays at dose range of 0.1 to $4.0 \mathrm{~Gy}$, followed by culturing them for 48-72 hours at $37{ }^{\circ} \mathrm{C}$ by the standard technique. After being harvested, the chromosome spread at metaphase and $\mathrm{MN}$ were stained with Giemsa's solution. The results showed that the frequency of both dicentrics and $\mathrm{MN}$ of samples were increased with the increase of radiation dose. Considerable increases of both cytologic damages were found in the samples exposed to higher doses ( $>2 \mathrm{~Gy}$ ). Significant differences $(p>0.05)$ only found in mean frequencies of $\mathrm{MN}$ for all doses tested. Reconstruction of the relationship of these frequencies with doses was found to follow linear-quadratic curve lines and was consistent with that of other studies. Due to the aforementioned advantages namely the dependence of radiation dose and dose rate on the frequency of of both dicentric and $\mathrm{MN}$, despite some limitations, these assays have been found to be suitable to be used as biological dosimetry. It is concluded that in order for this cytogenetic biodosimety method by means of scoring/assessing the radiation-induced dicentrics and MN could be used in radiation emergency and protection, and further studies with larger numbers of samples need to be done.
\end{abstract}

\section{INTRODUCTION}

For the benefit of humankind, radiation is widely used in many fields such as medicine, industry, and electricity. In addition, radiation has useful applications in agriculture, archaeology (carbon dating), space exploration, law enforcement,

\footnotetext{
* Corresponding author.

E-mail address: mukh_syaifudin@batan.go.id DOI: http://dx.doi.org/10.17146/aij.2017.624
}

geology, and many others. However, if the level of radiation is sufficiently hig, as in accident situations, it can be fatal for a human being. Organic materials such as human tissues could be damaged when they are exposed to ionizing radiation in form of either particles or electromagnetic waves [1-3]. An accurate and immediate irradiation dose assessment for human body must be conducted to allow for correct decision and appropriate choice of medical management, particularly in cases where 
the victims are suspected to have received high-dose irradiation exposures that may cause severe or lethal damages to human bodies [4].

In the cases of the radiation accident on occupational workers in irradiation control area, individual exposure dose is monitored either by area monitoring systems or by body-worn monitoring tools such as thermoluminescence dosimeters. However, the dose measured by these tools does not inform the actual damages occured in body. An even more problematic situation is those accidentally-exposed community members who do not usually wear these monitoring tools. Therefore, irradiation dose estimation in these situations must be performed by alternative methodologies such as biodosimetry $[4,5]$.

The effects of ionizing radiation on genetic material are well known. Double-strand deoxyribonucleic acid (DNA) breaks appear to be the primary lesions in the formation of chromosomal aberrations, which can easily be seen in metaphasic chromosomes [6]. One of these is the dicentric chromosome, which is a product of genome rearrangement that places two centromeres on the same chromosome [7]. Dicentric chromosomes have been identified as initiators of the genome instability associated with cancer, but this instability is often resolved by one of a number of different secondary events. These events include centromere inactivation, inversion, and intercentromeric deletion. It means that dicentric chromosomes are a well-known feature of cancer cells, and the induced genome instability and evolution are highly relevant to cancer biology [8]. Many of these dicentrics are also associated with birth defects such as Turner and Down Syndromes and with reproductive abnormalities. Although dicentrics can occur between any two chromosomes, some types are more prevalent than others in the human population.

The power of dicentrics for dose estimation is related to the low and constant spontaneous dicentric rate in the healthy population $[9,10]$. However, this assay is time consuming and highly technique dependent. In a mass-casualty situation, this assay is not well suited for providing timely dose estimates. It is very specific for radiations where background level for dicentrics in the population is low (about 1 dicentric chromosome in 1000 metaphase cells). A few chemicals also cause dicentrics. The dicentric assay is very sensitive to radiation; threshold doses of as low as about 0.05 Gy may be determined by this assay [11].

Besides dicentric chromosome aberrations, micronuclei (MN) examination also plays an important role in biological dosimetry. This technique is useful for determining dosage by examining a large number of binucleated cells and it is much faster and simpler than the chromosomal aberrations technique. The micronucleus is a byproduct of chromosomal aberrations in the form of a small circle in the cytoplasm outside the main nucleus and contains the fused chromosomes or its fragments, and/or chromosomes that are intact and appear with the same structure with the main core. Micronuclei formation is strongly influenced by radiation dose rate and also depends on the capacity of DNA and cellular repair $[9,12,13]$. Micronuclei disappear with a half-life of around one year and, though it is not specific to radiation exposure, has been used by several researchers to determine the radiation dose during radiotherapy or accidental radiation protection to ensure the program runs well. Thus, scoring the MN frequency is an alternative to the gold-standard dicentric assay for radiation biodosimetry in mass-casualty events.

Biological dosimetry or biodosimetry is a dose assessment method by means of observation of the symptoms or phenomena appearing after irradiation. Biodosimetry has become the standard test for dose assessment in the framework of radiological protection programmes. It allows dose estimation of an accidentally exposed person by comparing the observed aberration yield of dicentrics and $\mathrm{MN}$ to an in vitro calibration curve [9, 14-16]. This study had two aims, namely: first, to assess the effect of gamma-ray exposure on the frequency of dicentric chromosomes and $\mathrm{MN}$; and second, to generate standard curves as function of doses, to be used as radiation biodosimetry for predicting excessive radiation dose received by an individual. The aforementioned assessment would be expected to provide effective medical triages which have a potential to save tens of thousands of lives.

\section{EXPERIMENTAL METHODS}

\section{Research subjects}

This preliminary study enrolled seven healthy individuals (four Koreans and three Indonesians) of both sexes with an average age of 38.9 years (two females and five males). The subjects were non-smokers with no prior history of cancer. Every subject must fill out the informed consent form (willingness to provide blood samples) and their history of illness in last six months. Those biological samples were collected by using syringes and immediately put into vacuette tubes containing heparin (BD Vacutainer systems). Blood samples 
were irradiated in vitro with ${ }^{60} \mathrm{Co}$ gamma rays in acute whole body exposure, with seven different doses $(0.0 ; 0.1 ; 0.25 ; 0.5 ; 1.0 ; 2.0 ;$ and $4.0 \mathrm{~Gy})$ at a dose rate of $3.96 \mathrm{~Gy} / \mathrm{min}$. Irradiation was done in Gamma-cell 3000 Elam, Nordion International, Canada machine located at KIRAMS, Seoul, Korea. The assessment of dicentric chromosomes and MN for radiation biodosimetry was then performed.

\section{Culture setup and harvest for aberration analysis}

The analysis followed a standard procedure given by the International Atomic Eenegy Agency (IAEA) with slight modifications [10,17]. Two milliliters of the whole blood samples were cultured for $48 \mathrm{~h}$ in an incubator at $37{ }^{\circ} \mathrm{C}$ with a humid atmosphere of $5 \% \mathrm{CO}_{2}$. The culture medium consisted of $8.0 \mathrm{~mL}$ of RPMI-1640 supplemented with $10 \%$ heat-inactivated fetal calf serum and $1 \%$ streptomycin/penicillin (Gibco). Into this solution, $3.0 \% \mathrm{~mL}$ of phytohemagglutinin (Gibco BRL, Grand Island, NY) was added to stimulate cell division. Colcemid (Gibco BRL) was added for the last $4 \mathrm{~h}$ of culture at a final concentration of $0.1 \mathrm{mg} / \mathrm{mL}$ to block the mitotic process of the cells at the first metaphase stage. The content of each tube was then transferred into $15-\mathrm{mL}$ centrifuge tubes. The tubes were then centrifuged for ten minutes at $1500 \mathrm{rpm}$. The precipitate was re-suspend in $8 \mathrm{~mL}$ of $0.075 \mathrm{M} \mathrm{KCl}$ (pre-warmed to $37{ }^{\circ} \mathrm{C}$ ) for twenty minutes, which was followed by addition of $2 \mathrm{~mL}$ of cold fresh Carnoys Fixative (3:1 methanol:glacial acetic acid mixture). This fixation step was repeated two times (until white sediment was obtained). The yield of metaphasic cells was stored in freezer for at least one night until the preparation of slide was made.

\section{Scoring the metaphases}

Two to five slides were prepared for each sample, encoded, and then stained with $10 \%$ Giemsa's solution (Merck) and mounted. The number of aberrations was observed under a microscope (Nikon Eclipse Japan) connected to Olympus Camera System. By using the $100 \times$ oil immersion objective, a cell was considered as aberrant if it had one or more dicentric chromosome from each culture. Scoring was done by a single scorer in complete metaphase with more than 46 centromeres only as per the scoring criteria described in an IAEA Technical Report [10]. At least 2000 first division metaphasic cells were scored per sample. In the control samples,
3000-4000 metaphasic cells were analyzed per donor.

\section{Culturing and harvesting of lymphocytes for micronuclei}

Forty-four hours after the start of the culture, $15 \mu \mathrm{L}$ of cytochalasin B ( $3 \mathrm{mg} / \mathrm{mL})$ (Sigma) was added to the culture in a culture tube, and then MN were harvested 28 hours later $[10,18]$. Micronuclei harvesting was carried out by centrifugating the cultured blood at $1500 \mathrm{rpm}$ for 10 minutes followed by removal of the supernatant. A cold hypotonic solution $(8 \mathrm{~mL}$ of $0.075 \mathrm{M} \mathrm{KCl})$ was then added to the precipitate, which was then left at room temperature for three minutes. Formaldehyde (3-4 drops) and cold fixative solution (3:1 methanol:glacial acetic acid mixture, $7 \mathrm{~mL}$ ) were subsequently added, and the mixture was then mixed properly and placed in refrigerator $\left(4^{\circ} \mathrm{C}\right)$ for 10 minutes and was then centrifuged at $1000 \mathrm{rpm}$ for 10 minutes. The supernatant was removed and $6 \mathrm{~mL}$ of cold fixative solution was then added, which was followed by centrifuging at $1000 \mathrm{rpm}$ for 10 minutes. Binucleated cells (BNCs) which may contain $\mathrm{MN}$ will be obtained after three rounds of fixation process. The BNCs were then stored in a freezer for at least one night and $\mathrm{MN}$ was prepared by putting 3-4 drops of BNCs that contained $\mathrm{MN}$ on glass slide and allowed to dry in the air. The MN were stained with $4 \%$ Giemsa's solution and covered with cover glass and was observed under the microscope with a magnification of 1000 times. The MN counting in 1000 binuclear lymphocytes of each individual was done according to the criteria given in a standard protocol [10].

\section{Reconstructing the curve}

A dose-effect calibration curve for the yield of dicentrics and $\mathrm{MN}$ for ${ }^{60} \mathrm{Co}$ gamma rays in the 0 to 4.0 Gy range, using the maximum likelihood linear-quadratic model, $Y=c+\alpha D+\beta D^{2}$, was constructed with Dose Estimate software program (Version 5.1) and $U$-test according to A.A. Edwards et al. [19] to assess the uniformity of the radiation exposure distribution.

\section{RESULTS AND DISCUSSION}

Based on the chromosomal aberration assay in this report, we observed the higher frequencies of dicentrics chromosome in the exposed group (0.1-4.0 Gy) than in the control group (0 Gy, 
unexposed samples). Also found were three dicentrics in control samples (Table 1). In all samples, we also observed significantly higher MN frequencies per 1000 binucleated cells than in control, particularly samples that was irradiated with dose higher than $2.0 \mathrm{~Gy}$. On the other hand, at doses of 0.1-0.25 Gy there was significantly less cytogenetic damage. Our results indicate that there is a direct influence of dose on the frequency of chromosomal damage either as dicentric chromosome or MN. More lymphocytes with multiple MN (up to 4) were observed in samples received higher doses of radiation (Table 2). The development of $\mathrm{MN}$ implies substantial chromosomal damage and rearrangement. Doublestrand breaks of DNA are well-known precursors for most irradiation-induced MN.

The reconstructed of curve on the dose-effect relationships of cells exposed to ionizing radiation is described by a linear quadratic (LQ) model over an extended dose range (up to 4.0 Gy), which then plateaus for high doses (Fig. 1 and Fig. 2). The result of the equation showed that the value of $\alpha$ and $\beta$ for dicentrics are 0.0563 and 0.0057 , respectively, and for MN they are 0.1014 and 0.0105 , respectively. The correlation coefficient $(r)$ is between 0.993 and 1.21, indicating a goodness of fit of the calibration curve to the experimental data.

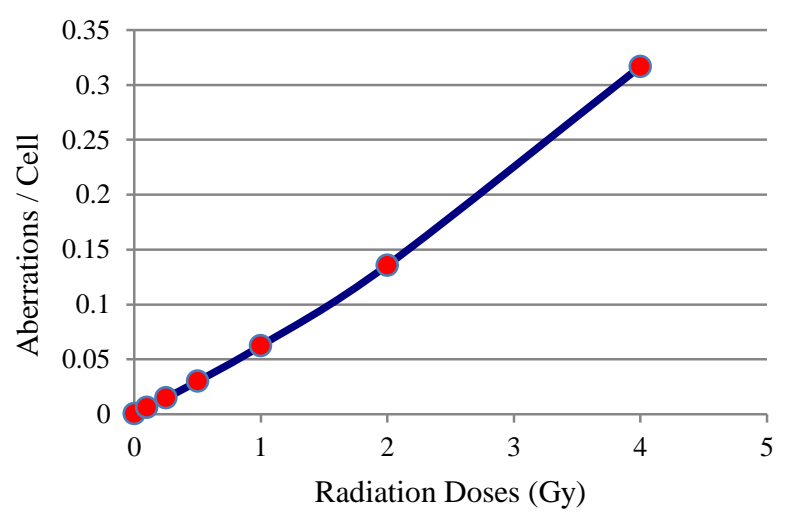

Fig. 1. Linear-quadratic curves representing the relationship between dose of irradiation and frequency of dicentrics.

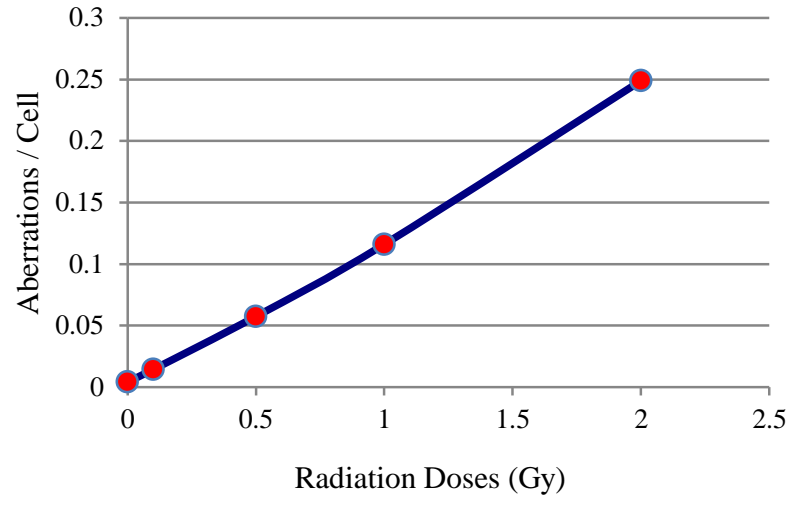

Fig. 2. Linear-quadratic curves representing the relationship between dose of irradiation and frequency of $\mathrm{MN}$.

As seen in Table 1, the distribution of dicentric chromosomes $(U)$ showed overdispersion for higher dose of irradiation $(0.5,2.0$, and $4.0 \mathrm{~Gy})$, indicating a heterogeneous (partial) exposure, except for $1 \mathrm{~Gy}$ that did not result in overdispersion (Poissonian). In Table 2 it can also be seen that all distribution of $\mathrm{MN}$, which were observed in the cytochalasine-B blocked cells, tend toward over dispersion when tested for conformity with the Poisson distribution using the Papworth's $U$ test. Due to the values of $u$ exceeded 1.96, the result was considered statistically significant at the level of $5 \%$. However, there is no data for $\mathrm{MN}$ for $4 \mathrm{~Gy}$ due to technical error.

These frequencies of dicentrics and $\mathrm{MN}$ obtained were used to construct dose-response curves to estimate absorbed radiation doses; to do so, seven different radiation doses ( 0.00 to $4.00 \mathrm{~Gy})$ were used. The results are presented in Figs. 1 and 2. Included here are four dose points at low doses between control ( 0 Gy) and 1.0 Gy dose range at which most of the possible radiation accidents occur [20]. At very low doses, the dependence of these cytogenetic damages (dicentric and $\mathrm{MN}$ ) on dose was fitted by a linear regression with a zero intercept. And in the dose range of about 0.5-1.0 Gy, there are plateaus, and above this range (more than 2 Gy) the curve again appears linear but with a different slope. The relationships between chromosomal aberrations and radiation were best expressed with the linear quadratic equation.

Table 1. Frequency $(Y)$ and distribution of dicentric chromosome induced by gamma rays at doses of up to 4.0 Gy in lymphocytes of peripheral blood samples from seven respondents.

\begin{tabular}{|c|c|c|c|c|c|c|c|c|c|c|c|}
\hline \multirow{2}{*}{$\begin{array}{l}\text { Dose } \\
\text { (Gy) }\end{array}$} & \multirow{2}{*}{$\begin{array}{c}\text { No. of } \\
\text { counted cells }\end{array}$} & \multirow{2}{*}{$\begin{array}{l}\text { No. Cells with } \\
\text { dicentrics }\end{array}$} & \multicolumn{6}{|c|}{ No. lymphocytes containing dicentrics } & \multirow{2}{*}{$Y \pm \mathrm{SE}$} & \multirow{2}{*}{$\sigma^{2} / y \pm \mathrm{SE}$} & \multirow{2}{*}{$U$} \\
\hline & & & 0 & 1 & 2 & 3 & 4 & 5 & & & \\
\hline 0.0 & 4000 & 3 & 3997 & 3 & 0 & 0 & 0 & 0 & $0.001 \pm 0.000$ & $0.999 \pm 0.018$ & -0.027 \\
\hline 0.1 & 3500 & 27 & 3473 & 27 & 0 & 0 & 0 & 0 & $0.008 \pm 0.001$ & $0.993 \pm 0.023$ & -0.317 \\
\hline 0.25 & 4000 & 10 & 3990 & 10 & 0 & 0 & 0 & 0 & $0.003 \pm 0.001$ & $0.998 \pm 0.021$ & -0.106 \\
\hline 0.5 & 4000 & 85 & 3924 & 68 & 7 & 1 & 0 & 0 & $0.021 \pm 0.002$ & $1.21 \pm 0.022$ & 9.64 \\
\hline 1.0 & 4000 & 257 & 3755 & 233 & 12 & 0 & 0 & 0 & $0.064 \pm 0.004$ & $1.030 \pm 0.022$ & 1.32 \\
\hline 2.0 & 3500 & 626 & 2936 & 511 & 44 & 9 & 0 & 0 & $0.179 \pm 0.007$ & $1.050 \pm 0.024$ & 2.02 \\
\hline 4.0 & 1853 & 505 & 1348 & 344 & 59 & 13 & 1 & 0 & $0.273 \pm 0.014$ & $1.14 \pm 0.033$ & 4.26 \\
\hline
\end{tabular}


Table 2. Frequency $(Y)$ and distribution of MN induced by gamma rays at doses of up to 2 Gy for lymphocytes from blood samples obtained from seven subjects.

\begin{tabular}{|c|c|c|c|c|c|c|c|c|c|c|c|}
\hline \multirow{2}{*}{ Dose (Gy) } & \multirow{2}{*}{$\begin{array}{l}\text { No. cells } \\
\text { counted }\end{array}$} & \multirow{2}{*}{$\begin{array}{l}\text { Total no. } \\
\text { of MN }\end{array}$} & \multicolumn{6}{|c|}{ No. cells with MN } & \multirow{2}{*}{$Y \pm \mathrm{SE}$} & \multirow{2}{*}{$\sigma^{2} / y \pm \mathrm{SE}$} & \multirow{2}{*}{$U$} \\
\hline & & & 1 & 2 & 3 & 4 & 5 & Total & & & \\
\hline 0.0 & 3000 & 15 & 11 & 2 & 0 & 0 & 0 & 13 & $0.005 \pm 0.001$ & $1.260 \pm 0.025$ & 10.5 \\
\hline $\left.0.1^{*}\right)$ & 2000 & 18 & 14 & 2 & 0 & 0 & 0 & 16 & $0.009 \pm 0.002$ & $1.210 \pm 0.031$ & 6.96 \\
\hline 0.5 & 3000 & 165 & 121 & 12 & 4 & 2 & 0 & 139 & $0.055 \pm 0.006$ & $1.380 \pm 0.026$ & 14.8 \\
\hline 1.0 & 3000 & 375 & 201 & 66 & 10 & 3 & 0 & 280 & $0.125 \pm 0.009$ & $1.480 \pm 0.026$ & 18.7 \\
\hline 2.0 & 3000 & 734 & 330 & 130 & 40 & 6 & 0 & 506 & $0.245 \pm 0.014$ & $1.540 \pm 0.026$ & 20.7 \\
\hline
\end{tabular}

*) Note : cells were counted from 2 samples.

Figure 3 represent two Giemsa-stained dicentric chromosomes seen in a metaphasic cell after irradiated with 2.0 Gy in a subject. These two dicentric, according to our experince, are commonly induced by dose higher than $1.0 \mathrm{~Gy}$. This dicentric chromosome is a well-known feature of cancer cells, and the genome instability and evolution it induce are highly relevant to cancer biology.

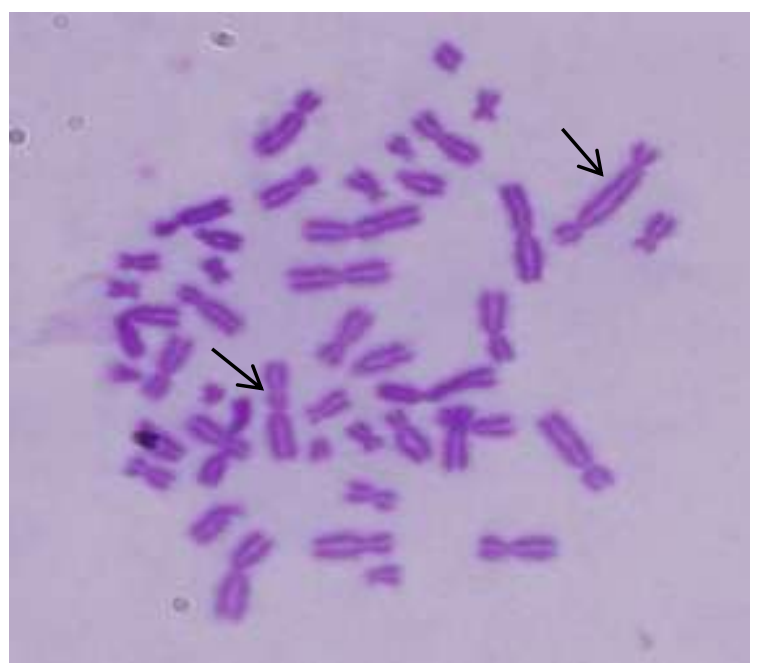

Fig. 3. A Giemsa-stained metaphasic cells containing two dicentrics (arrows) in lymphocytes of the whole blood irradiated with 2 Gy of gamma rays.

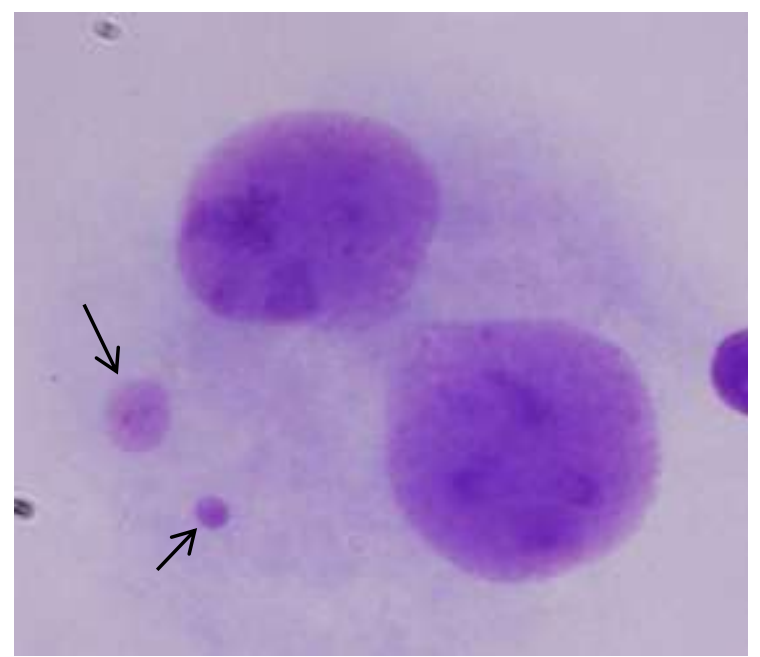

Fig. 4. Cells containing two MN (arrows) beside binucleated cell (BNC) surrounded by cytoplasm after irradiation with 2 Gy of gamma rays.
Furthermore, Fig. 4 showing two MN formed beside a BNC after being irradiated with a dose of $2.0 \mathrm{~Gy}$. It can be seen that two main nuclei in a BNC may touch, but ideally should not overlap each other, and equal in size, staining pattern, and staining intensity. They are also scored for peripheral blood lymphocytes in the first interphase after cell division.

The relationship between radiation dose and biological effects obtained was similar with the results of other studies. Research conducted by Lemos-Pinto et al. [21] showed that the yield of dicentrics increased with radiation dose of $6 \mathrm{MV}$ electron linear accelerator. These dose-dependent results clearly indicated that the data well represented by the linear-quadratic model based on CABAS and Dose Estimate software programs. Cho et al. [22] also evaluated the cumulative gamma-radiation personal exposure by analysing translocations using FISH painting and found a linear quadratic relationship between dose of radiation and translocation. Furthermore, Ulsh et al. [23] reconstructed a relationship of dose-effects obtained from 13 individuals who were exposed to Cobalt-60 in a Thailand accident. Pujo et al. [24] also found a linear-quadratic model of dose-effect curve assuming the Poisson distribution, and some others $[12,15,19]$.

In addition to precise dose reconstructions, biodosimetry can also be used in the immediate response to accidents, where few cells need to be scored initially, and for medical triage of either whole-body or partial-body irradiation. In such cases, it would play an important role in national emergency responses to a large-scale accident where many people may have been exposed.

In the present study, cellular radiation responses to damage caused by gamma rays have been investigated in lymphocytes from seven human subjects. The number of subjects was very limited and were not necessarily representative of the Indonesian or Korean population. Besides, all samples were obtained from adults, and did not include younger subjects (teenage and children) that may be involved in emergency situation. In order to obtain more representative samples, further studies 
with larger number and wider age-range groups of subjects need to be performed.

Scoring of unstable chromosomal aberrations (dicentrics, rings, and fragments) in circulating lymphocytes is the most extensively studied biological means for estimating individual exposure to ionizing radiation [25,26]. Dicentrics are a biological marker that is specific to radiation injury; it is the gold standard of biodosimetry and can also reveal partial-body exposures. Therefore, our study was focused only on this biological marker. The simplicity, rapidity and sensitivity of the cytokinesis-block MN assay makes it a valuable tool for screening. Additionally, the multiple endpoints simultaneously generated lead to a better understanding of the underlying mechanisms involved in the carcinogenic process that in turn could substantially improve risk predictions. The assay's reliability and low cost are other reasons that this method is the most frequently used one for the assessment of in vitro chromosomal radiosensitivity and cancer risk among the cytogenetic assays [27]. It has a lower detection limit of about $0.1 \mathrm{~Gy}$, and allows one to distinguish between whole- and partial-body exposures.

The main advantages of scoring dicentrics for biodosimetric evaluations are their high radiation specificity, low background in non-exposed individuals (0-1 dicentric per 1000 cells), low intervariability, and low detection limits of 0.1 Gy for low linear-energy-transfer radiation. Its reliability and validity can be further improved by confirming the dicentric results obtained by the labs from the Giemsa-stained metaphase preparations with centromere-specific FISH [28]. Various cytogenetic end-points, including counting chromosomal aberrations and $\mathrm{MN}$, have also been previously utilised as biomarkers of cancer susceptibility in non-carriers [29].

This work was designed to investigate the use of both methodologies (scoring of dicentrics and $\mathrm{MN}$ ) for evaluation of dose from whole-body exposure, in which they would give different results from what would be obtained from their application to the partial-body exposure to ionizing radiation. This was possible due to the characteristics of treatment and health conditions of each subject. The risk of harm from radiation is largely dependent upon such factors as the dose of irradiation, the rate at which it was delivered, the type of radiation, the part of the body exposed, and the age and health as well as the sex of the exposed individual $[17,30]$.

Accurate dosimetry, the quantitative determination of radiation energy absorbed into the body, is a necessary prerequisite for practically all applications of ionizing radiation in daily life. Blood cell count, such as circulating leukocytes or lymphocyte that are very sensitive to ionizing irradiation, is a good indicator for the exposed dose [31]. Lymphocyte or leukocyte counts decrease after irradiation, then they usually recover, but will not increase or never return after high-dose exposures. This is also make it difficult for calculate the given dose by the kinetics of hematopoietic cell data. Other dose assay protocols using newer molecular biology biomarker method have recently been developed, such as $\gamma \mathrm{H} 2 \mathrm{AX}$ detection after DNA damage [32,33]. The use of these multiple assays could help reducing the uncertainties that arise from inter-individual and intra-individual variabilities.

Biological dosimetry using the analysis of unstable chromosomal aberrations in human lymphocytes from peripheral blood is well established and accurate, especially in the case of acute exposure, and when the blood samples are obtained within few days after the real or suspected radiation exposure [23]. An interlaboratory comparison that validates the dicentric chromosome assay for assessing radiation dose in mass casualty accidents is needed to identify the advantages and limitations of an international biodosimetry network.

There are factors that influence the observed frequencies of $\mathrm{MN}$ and also known to have an impact on the resulting calibration curves, such as differences in the lymphocyte donors and culture protocols, slide preparation, and scoring criteria [25,34]. Therefore, to increase the accuracy of dose estimation, each laboratory should have its own calibration curve. Moreover, such factors as the type of radiation, energy, and dose rate employed, all directly influence the values of $\alpha$ and $\beta$, considering the respective relative biological effectiveness (RBE) of different energies for producing dicentric chromosomes [35]. The type of sample required, dose detection limit, time interval when the assay is feasible biologically, time for sample preparation and analysis, and ease of use also have to be considered.

From the previous discussion, it is understood that cytogenetic biodosimetry is a simple, useful, and unique irradiation dose assessment method for human body and is very important both for dose estimation during urgent irradiation exposure accidents and for evaluating chronic or repeated exposure and assess late irradiation effect [36]. Chromosomal aberrations indicate actual damages occuring in cells or organs by both external or internal irradiation exposure, which is unique, different from other physical or chemical methods. 


\section{CONCLUSION}

The frequencies of both dicentrics and micronuclei after irradiation of lymphocytes increased with the increasing radiation dose, mainly for higher doses (>2 Gy). Significant differences $(p>0.05)$ or overdispersion only found in mean frequencies of $\mathrm{MN}$ for all doses tested. Reconstruction of the relationship of these frequencies with doses followed a linear-quadratic curve lines, and therefore it is very important for dose estimation in radiation emergency and for evaluate or assess irradiation effect.

\section{ACKNOWLEDGMENT}

This work was financially supported by the 2014 fiscal year Indonesian Governmental Annual Research Project (DIPA) of the Center for Radiation Safety Technology and Metrology, and Annual Project of Laboratory of Radiation Cytogenetics and Epidemiology, Korea Institute of Radiological and Medical Sciences (KIRAMS), South Korea.

\section{REFERENCES}

1. Anonymous, Health Risks From Exposure to Low Levels of Ionizing Radiation, National Research Council. BEIR VII, The National Academy Press, Washington DC. (2006) 45.

2. C.N. Coleman, C. Hrdina, J.L. Bader et al., Ann. Emerg. Med. 53 (2009) 213.

3. G.A. Nyberg, D. Stricklin and A. Sellstrom, Int. J. Environ. Res. Public. Health. 8 (2011) 4521.

4. D.M. Christensen, C.J. Iddins and S.L. Sugarman, Emerg. Med. Clin. North. Am. 32 (2014) 245.

5. J.M. Sullivan, P.G. Prasanna, M.B. Grace et al., Health Phys. 105 (2013) 540.

6. P H. Romm, E. Ainsbury, S. Barnard S et al., Health Phys. 106 (2014) 764.

7. K.M. Stimpson, J.E. Matheny and B.A. Sullivan, Chromosome Res. 20 (2012) 595.

8. Z. Shen, J. Mol. Cell. Biol. 3 (2011) 1.

9. A. Vral, M. Fenech and H. Thierens, Mutagenesis 26 (2011) 11.

10. Anonymous, Technical Reports Series 405, IAEA, Vienna (2011).
11. F.N. Flegal, Y. Devantier, J.P. McNamee et al., Health Phys. 98 (2010) 276.

12. J.D. Tucker, M. Vadapalli, M.C. Joiner et al., Radiat. Res. 180 (2013) 284.

13. E. Pernot, J. Hall, S. Baatout et al., Mutation Res. 751 (2012) 258.

14. Y. Xu, H.C. Turner, G. Garty et al., Biomed. Eng. Res. 2 (2013) 16.

15. K. Al-Hadyan, S. Elewisy, B. Moftah et al., Biotech. 4 (2014) 635.

16. W.F. Blakely, C.A. Salter and P.G. Prasanna. Health Phys. 89(5) (2005) 494.

17. D.J. Blumenthal, S.L. Sugarman, D.M. Christensen et al., Health Phys. 106 (2014) 516.

18. K. Shirsath, N. Bhat, K. Anjaria et al., Int. J. Radiat. Res. 12 (2014) 211.

19. A.A. Edwards, D.C. Lloyd, R.J. Purrott, Radiat. Environ. Biophys. 16 (1979) 89.

20. S.A. Haeri, H. Mozdarani, M. Foroghizadeh et al., Iran. J. Radiat. Res. 2 (2004) 85.

21. M.M.P. Lemos-Pinto, M. Cadena, N. Santos et al., Braz. J. Med. Biol. Res. 48 (2015) 945.

22. M. S. Cho, J. K. Lee, K. S. Bae et al., J. Radiat. Res. 56 (2015) 709.

23. B.A. Ulsh, J. Dolling, J. Lavoie et al., Dose Response 13 (2015) 1.

24. M. Pujo, J.F. Barquinero, P.Puig et al., PLoS One. 9 (2014) e114137.

25. P.G.S. Prasanna, M. Moroni, and T.C. Pellmar, Health Phys. 98 (2010) 244.

26. A. Wojcik, G. Stephan, S. Sommer et al., Radiat. Res. 160 (2003) 677.

27. Y. Zhao, P. Xie and H. Fan, Environ. Sci. Technol. 46 (2012) 34.

28. R. El-Zein, A. Vral1 and J.C. Etzel, Mutagenesis 26 (2011) 101.

29. H. Romm, E. Ainsbury, S. Barnard et al., Health Phys. 106 (2014) 764.

30. P. Boffetta and F. Islami, Ann. Oncol. 24 (2013) 901.

31. Q. Zhang, B. Yang, X. Zhai et al., Int. J. Pharmacol. 10 (2014) 524.

32. C.E. Redon, J.S. Dickey, A.J. Nakamura et al., $H 2 A X$ in DNA Damege Response, in: Molecular Determinants of Radiation Response Vol. I, T.L. De Weese and M. Laiho (Eds.), Springer, New York (2011) 3. 
33. S.E. Polo and S.P. Jackson, Genes \& Dev. 25 (2011) 409.

34. J.L. Huret, M. Ahmad, M. Arsaban et al., Nucleic. Acids. Res. 41 (2013) D920.
35. J.M. Sullivan, P.G.S. Prasanna, M.B. Grace et al., Health Phys. 105 (2013) 540.

36. H. Romm, E. Ainsbury, S. Barnard et al., Mutat. Res. 756 (2013) 174 